\title{
Dividing a Circular Arc into Equal Number of Divisions
}

\author{
Dr A. M. Chandra \\ (Department of Civil Engineering, Sharda, University, India)
}

\begin{abstract}
No manual method exists to divide a circular arc into equal numbers of division. This paper presents a method of dividing a circular arc into equal numbers of division using the method of arc rectification. The manual method of dividing a circular arc presented in this paper is completely a new method developed by the author, and will be very useful in graphics.
\end{abstract}

Keywords: Circular arc, Chord, Bisection, Rectification

\section{Introduction}

In the book Engineering Graphics (Chandra, et al., 2003), to construct a scale of chords, a circular arc is to be divided. By oversight, we forgot to write the method of dividing the circular arc into nine (odd) equal numbers of divisions. This omission was found by Mr. John Timko of the Penn State University, U.S.A., when he wanted to use the book for teaching the course, and the same was communicated to me as he wanted to know the method of dividing the arc. After knowing the omission, it was realized that no such method exists except to divide the arc by trial and error method. The author of this paper, then thought to develop a method of dividing a circular arc in odd equal numbers of division based upon some solid foundation, and the same is presented as author's own work.

\section{Existing Method}

The existing method which can be used to divide a circular arc in even equal numbers of divisions, is the method of successive bisections. In Fig. 1, the arc $A B$ is divided into two parts by dividing the chord $A B$ in two equal parts as $A a$ and $a B$, then locating $C$ on the arc by drawing a perpendicular to $A B$ at its midpoint $a$, and then extending the perpendicular to intersect the $\operatorname{arc} A B$ at $C$. Now the same procedure is applied to divide the arcs $A C$ and $C B$, each in two equal parts, thus dividing the arc $A B$ in four equal parts. The points $D$ and $E$ are located by drawing perpendiculars on $A C$ and $C B$ at $b$ and $c$, respectively, and so on.

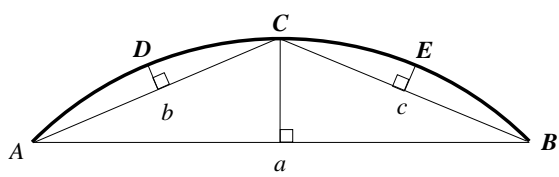

Fig. 1 Successive bisection method

When the arc is to be divided into odd equal numbers of part, the method of successive bisections cannot be used. The only method available is trial and error method. Set a convenient distance $d_{1}$ by judgment on a bow divider as show in Fig. 2, and mark the points 1, 2, 3, etc., on the arc. Let the last point 9 be outside the arc. Now by judgment reduce the distance $d_{1}$ to $d_{2}$, and mark another set of points $1^{\prime}, 2^{\prime}, 3^{\prime}$, etc. Let the last point, in this trial, be at $9^{\prime}$. In the third trial, $d_{2}$ is slightly increased by judgment, and the points are again marked on the arc. The process is continued till the last point 9 falls on the point $B$.

In the first trial, as shown in Fig. $2 \mathrm{~b}$, the last point may fall at 9, i.e., within the arc, and in such cases, the second trial will be made by increasing the distance $d_{1}$.
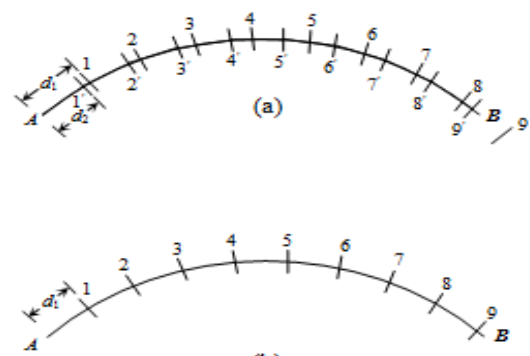

(b)

Fig. 2 Trial and error method 


\section{Author's Method Of Rectification}

The method developed by the author, is based on the principle of rectification of arc. The rectification is a process of converting a circular arc into an equivalent straight line. Once an arc is in the form of a straight line, the straight line can be divided into desired number of equal parts by usual method of drawing parallel lines. After locating the points on the straight line, the length of one division is known which can be used to divide the arc.

Let $A B$ be the circular arc that is to be divided into, say seven numbers of equal parts (Fig. 3). The arc $A B$ is first rectified as $B A^{\prime}$. To rectify the arc $A B$, adjust the bow divider for a convenient distance, and beginning at $A$, set off equal distances $A a, a b, b c$, etc., until the division point $e$ nearest to $B$ is reached. At this point, reverse the direction, and set off an equal number of distances along the tangent $B T$ to the arc at $B$, to determine the points $e^{\prime}, d^{\prime}, \ldots \ldots, A^{\prime}$ on the tangent. Draw a line $B C$ at any convenient angle to $B T$, and divide it into seven equal parts using a bow divider marking the divisions as $1^{\prime}, 2^{\prime}, \ldots \ldots, 7^{\prime}$. Join the point $7^{\prime}$ and $A^{\prime}$, and draw the line $6^{\prime}-a^{\prime \prime}$ parallel to the line $7^{\prime}-A^{\prime}$. Measure the distance $A^{\prime} a^{\prime}$ on the bow divider, and mark the points 1 , $2, \ldots \ldots, 6$ on the arc taking $A-1,1-2, \ldots \ldots, 6-B$, equal to $\mathrm{A}^{\prime} \mathrm{a}^{\prime \prime}$. Thus the $\operatorname{arc} A B$ is divided into seven equal parts.

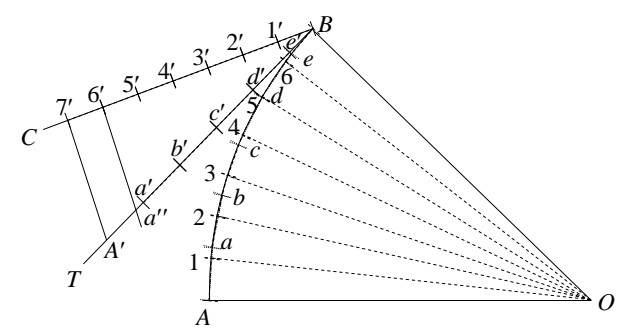

Fig. 3 Dividing a circular arc in equal numbers of part by rectification

The above constructions are approximate, but well within the range of accuracy of drawing instruments. For rectification of the arc, the last point $e$ should be as close to $B$ as possible, i.e., $e B$ should be as small as possible. The smaller are the divisions for rectification, better would be the accuracy of rectification. The rectification length $B A^{\prime}$ is slightly shorter than the length of the arc $A B$. If the angle subtended by each division is $10^{\circ}$, the error is approximately 1 in 830 .

\section{Conclusions}

Since no other method is available to manually divide an arc into odd equal numbers of part, the method developed is the only method which can be used to divide an arc manually in equal odd or even numbers of part. This method is faster than the trial and error method as it does not require trials which consume lot of time. The accuracy of division can be increased by taking smaller divisions at the time of rectification.

\section{Reference}

[1] Chandra, A. M. and Chandra, S. (2003): Engineering Graphics, Narosa Publishing House, New Delhi, $2^{\text {nd }}$ ed., PP. 65-66, 2003. 